\title{
Estimations de la prévalence des troubles bipolaires traités par les services de santé mentale : utilisation de données administratives de l'Alberta et d'enquêtes sanitaires pancanadiennes
}

\author{
A. G. Bulloch, Ph. D. (1); S. Currie, Ph. D. (2); L. Guyn, B. Sc., B.A. (2); J. V. Williams, M. Sc. (1); D. H. Lavorato,
} M. Sc. (1); S. B. Patten, M.D., Ph. D. (1)

\section{Résumé}

Introduction : Des estimations précises de la charge de morbidité sont nécessaires pour offrir des services de santé mentale adaptés à la population.

Méthodes : Nous avons évalué la prévalence des cas de trouble bipolaire traités par les services de santé mentale dans la zone de Calgary, circonscription hospitalière albertaine comptant une population de plus d'un million d'habitants. Nous avons utilisé les données administratives conservées dans un dépôt central fournissant les renseignements sur les contacts pour des soins de santé mentale d'environ $95 \%$ des services de santé mentale financés par l'État. Nous avons comparé cette prévalence des cas traités aux données autodéclarées de l'Enquête sur la santé dans les collectivités canadiennes : Santé mentale et bien-être de 2002 (ESCC 1.2).

Résultats : Sur les 63016 personnes âgées de 18 ans et plus traitées dans la zone de Calgary en 2002-2008, 3659 (5,81 \%) ont reçu un diagnostic de trouble bipolaire de type I et $1065(1,70 \%)$, de trouble bipolaire de type II. On estime que la prévalence des cas traités de ces troubles s'établit à 0,41 \% dans le premier cas et à 0,12\% dans le second. Nous avons estimé, d'après les données de l'ESCC 1.2, qu'entre 0,44 \% et 1,17 \% de la population canadienne était traitée par des psychiatres pour un trouble bipolaire de type I.

Conclusion : Dans le cas du trouble bipolaire de type I, l'estimation fondée sur les données administratives locales est proche de la borne inférieure de la fourchette de l'enquête sanitaire. Le degré de concordance dans nos estimations prouve l'utilité des dépôts de données administratives dans la surveillance des troubles mentaux chroniques.

Mots-clés : trouble bipolaire, données administratives, enquêtes sanitaires, prévalence, Alberta

\section{Introduction}

Il convient de disposer d'estimations précises de la charge de morbidité associée aux troubles mentaux dans la population pour être en mesure de fournir des services de santé mentale adéquats. En général, on utilise pour les estimations de la prévalence de troubles mentaux dans l'ensemble de la population des données tirées d'enquêtes sanitaires réalisées en personne ou par téléphone. Cependant, ces enquêtes sur la santé présentent un certain nombre de lacunes. Ainsi, l'Enquête sur la santé dans les collectivités canadiennes : Santé mentale et bien-être de 2002 (ESCC 1.2) ${ }^{1}$, qui évalue la prévalence des troubles mentaux et l'utilisation des services de santé, s'appuie sur des données autodéclarées plutôt que sur le diagnostic d'un professionnel. Ces données sont certes obtenues par un personnel qualifié, dans le cadre d'interviews directes, mais elles sont sujettes au biais de rappel, d'où l'intérêt éventuel d'estimations reposant sur d'autres sources de données.

Au Canada, le secteur public des soins de santé fournit la majorité des services de santé, y compris le traitement de la toxicomanie et des troubles mentaux. Des renseignements détaillés sur les bénéficiaires des services de santé sont saisis dans divers ensembles de données administratives. Il est facile d'y accéder, et leur utilisation aux fins de recherche est rentable ${ }^{2}$. Ces bases de données fournissent une perspective concrète du traitement des troubles mentaux, qui permet une généralisation de l'état de la prestation de services de santé mentale. En outre, les ensembles de données administratives peuvent fournir des estimations précises de la prévalence des cas traités et éviter le biais de rappel des enquêtes sanitaires ${ }^{3}$. Ils peuvent donc contribuer notablement à augmenter la capacité de surveillance de la santé à l'échelle nationale ${ }^{4}$.

On utilise des données administratives sur la santé mentale pour étudier les répercussions des changements apportés au système sur l'utilisation des services et sur la qualité des soins ${ }^{5}$, les variations dans les méthodes de traitement dans différents milieux de soins ${ }^{6}$, la mesure de la performance, y compris l'adhésion aux pratiques

Rattachement des auteurs :

1. Department of Community Health Sciences and Hotchkiss Brain Institute, Université de Calgary, Calgary (Alberta), Canada.

2. Mental Health Information Management, Evaluation \& Research, Alberta Health Services, Addiction \& Mental Health, Calgary Zone, Calgary (Alberta), Canada.

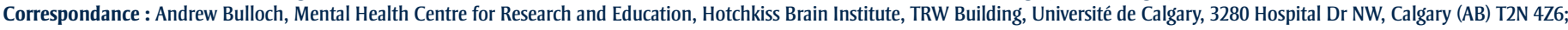
tél. : 403-220-4586; téléc. : 403-210-8840; courriel : bulloch@ucalgary.ca 
exemplaires ${ }^{7}$, les prédicteurs de l'utilisation des services ${ }^{8}$, ainsi que pour déterminer la proportion de l'ensemble de la population souffrant de troubles mentaux qui reçoit un traitement ${ }^{9,10}$ et le rapport coût-efficacité des services de santé mentale ${ }^{11}$. On les utilise aussi dans la recherche sur la santé d'une population locale ${ }^{2}$ et dans une évaluation à long terme de l'évolution de l'utilisation des services d'urgence psychiatrique ${ }^{12}$.

La zone de Calgary est l'une des cinq circonscriptions hospitalières définies pour la province de l'Alberta. Dans cette province, tous les services de santé publique relèvent d'un seul organisme appelé Alberta Health Services (AHS). La zone de Calgary couvre une région géographique de $39000 \mathrm{~km}^{2}$ et compte plus de 1,3 million d'habitants. Elle comprend une grande ville (Calgary) et plusieurs villes de plus petite taille, dont Banff, Airdrie, Okotoks et Canmore. La zone de Calgary offre un vaste éventail de services de santé mentale et de traitement de la toxicomanie pour adultes : traitements spécialisés avec hospitalisation dans trois grands hôpitaux urbains, services d'hôpital de jour, programmes de consultations externes, dont une clinique qui se spécialise dans les troubles bipolaires, et programmes d'approche dans la collectivité. Les personnes souffrant de trouble bipolaire peuvent s'adresser à tous ces services sans rien avoir à débourser personnellement.

L'utilisation d'un dépôt de données central créé en reliant les données administratives de différents systèmes d'information est une façon novatrice de calculer la prévalence, au cours d'une période déterminée, des troubles de santé mentale traités. Cette approche est différente de celle adoptée par la plupart des études reposant sur le couplage de dossiers au Canada, pour lesquelles les consultations chez le médecin de famille ou les hospitalisations constituent les principales rencontres avec les patients. Le dépôt de données de la zone de Calgary relie des données de tout l'éventail des services psychiatriques : programmes en hospitalisation, en hôpital de jour, en consultation externe et services d'approche.
Il est donc unique, même s'il ressemble effectivement à l'ancien système de couplage des dossiers psychiatriques de Kingston (Kingston Psychiatric Record Linkage System $)^{13}$. La majorité des travaux de recherche qui utilisent des données administratives portent sur les utilisateurs de services de soins de courte durée. Cependant, bien des gens atteints de troubles mentaux n'ont jamais besoin d'être hospitalisés ni de recevoir des soins psychiatriques d'urgence. Les données de facturation des médecins n'aident guère non plus à évaluer la prévalence de certains troubles mentaux. En effet, en Alberta, les médecins ne doivent indiquer que les trois premiers chiffres du code de la CIM-9* qui identifie par exemple le patient comme présentant un trouble de l'humeur de type dépressif ou de type bipolaire. De plus, le régime dit des Alternate Relationship Plans peut empêcher d'avoir accès aux données de la facturation des médecins, car il remplace la facturation à l'acte. Par exemple, dans un cadre multidisciplinaire, les médecins peuvent être rémunérés à la vacation aux termes d'un contrat qui n'exige pas de fournir un code de diagnostic dans la facturation des actes ou il se peut même qu'ils n'aient pas à fournir du tout de facturation des actes.

Les troubles bipolaires peuvent être dévastateurs. Ils apparaissent généralement à un jeune âge et sont associés à un risque élevé de suicide ${ }^{14}$. Le trouble bipolaire de type I est caractérisé par la survenue d'un ou de plusieurs épisodes maniaques ou épisodes mixtes qui peuvent s'accompagner d'un ou plusieurs épisodes dépressifs majeurs ${ }^{15}$. Les symptômes de l'épisode maniaque comprennent une fuite des idées ou la sensation que les pensées défilent, une augmentation de l'estime de soi, une réduction du besoin de sommeil, une plus grande communicabilité et une irritabilité. Le trouble bipolaire de type II est caractérisé par la survenue d'épisodes hypomaniaques qui, à la différence des épisodes maniaques, ne sont pas assez sévères pour entraîner une altération marquée du fonctionnement professionnel ou social ou pour nécessiter l'hospitalisation. Pour satisfaire aux critères diagnostiques du DSM-IV-TR ${ }^{\dagger}$ pour ce qui est du trouble bipolaire de type II, il doit aussi y avoir un ou plusieurs épisodes dépressifs majeurs.

On avance souvent que les troubles bipolaires sont sous-diagnostiqués, mais certains auteurs affirment le contraire ${ }^{16}$. Il a été proposé, mais c'est un sujet de controverse, d'abaisser le seuil de diagnostic du trouble bipolaire, ce qui donnerait des estimations de la prévalence sensiblement plus élevées ${ }^{17}$. De toute façon, il demeure nécessaire d'évaluer la prévalence telle qu'elle est mesurée aujourd'hui en fonction des cas traités.

Notre étude vise à comparer les estimations de la prévalence des cas traités de trouble bipolaire provenant de l'ESCC 1.2 et celles du dépôt de données des services de santé mentale de la zone de Calgary.

\section{Méthodes}

Cette étude repose sur des données provenant de deux sources. Les estimations nationales de la prévalence des cas traités de trouble bipolaire de type I dans l'ensemble de la population sont tirées de l'ESCC 1.2. Nous les avons comparées à la prévalence des cas traités de troubles bipolaires de type I et de type II calculée à partir des données administratives de la zone de Calgary. Pour ce qui est du type de médecin, les données administratives couvrent divers services de santé mentale, mais pas les médecins généralistes (MG). Afin de pouvoir comparer les deux ensembles de données, nous avons limité notre analyse de l'ESCC 1.2 aux seuls psychiatres.

\section{Enquête nationale sur la santé mentale}

L'ESCC 1.2 a été décrite en détail ailleurs ${ }^{18}$. Pour résumer, cette enquête menée en 2002 est une enquête ponctuelle en population visant à surveiller la santé mentale des Canadiens, ainsi que leurs besoins en matière de services de santé mentale et leur utilisation de ces services. Statistique Canada a obtenu un échantillon représentatif à l'échelle nationale de personnes âgées de 15 ans ou plus en 2002; cet échantillon ne comprenait aucune personne vivant dans 
les trois territoires, aucun membre des Forces armées, aucun Autochtone et aucune personne vivant en établissement ou dans des régions éloignées. Le taux de réponse a été de 77 \% ( $\mathrm{n}=36$ 984). Un personnel qualifié a mené les interviews : dans la majorité des cas, des interviews directes et lorsque cela n'était pas possible, des interviews téléphoniques.

Le Conseil de recherches en sciences humaines nous a autorisés à interroger le fichier principal de l'ESCC 1.2 , et nous avons consulté ces données au Centre de données de recherche régional des Prairies de Statistique Canada à l'Université de Calgary. Nous avons obtenu l'autorisation éthique nécessaire pour avoir accès aux données auprès du Conjoint Health Research Ethics Board de l’Université de Calgary.

L'évaluation du trouble bipolaire de type I dans l'ESCC 1.2 repose sur le diagnostic d'épisodes maniaques ou d'épisodes mixtes conformément aux critères diagnostiques du DSM IV-TR ${ }^{13}$. Les questions particulières sur les épisodes maniaques reposaient sur une version de World Mental Health de la Composite International Diagnostic Interview (WMH-CIDI) ${ }^{19}$ modifiée pour l'ESCC 1.2 et étaient posées par des intervieweurs qualifiés. Il n'était pas demandé aux répondants s'ils souffraient d'un trouble bipolaire : on leur posait plutôt une série de questions. Ensuite, des algorithmes ont été utilisés pour évaluer ce trouble en fonction des réponses données. Deux algorithmes ont été utilisés pour déterminer s'il y avait eu des épisodes maniaques au cours de l'année écoulée (prévalence sur 12 mois) ou dans la vie des répondants (prévalence au cours de la vie). Des questions différentes étaient posées selon qu'un MG ou un psychiatre traitait leur trouble.

Pour calculer la prévalence des cas traités de trouble bipolaire de type I dans la population canadienne, nous avons relié les données de l'ESCC 1.2 aux estimations de population avec des intervalles de confiance (IC) à $95 \%$. (Il convient de noter que l'ESCC 1.2 ne traite pas du trouble bipolaire de type II.) Ces estimations et les IC ont été pondérés et la méthode bootstrap a été employée, avec, pour compenser les procédures d'échantillonnage complexes, des poids d'échantillonnage et des poids de rééchantillonnage bootstrap fournis par Statistique Canada. Par exemple, les petites provinces ont été suréchantillonnées, de sorte que l'incidence de ces résultats sur l'estimation nationale doit être réduite en conséquence, c.-à-d. qu'il faut lui accorder moins de poids. Comme la taille de l'échantillon des cas de trouble bipolaire dans l'ESCC 1.2 était insuffisante pour établir une estimation séparée fiable pour la province de l'Alberta, nous avons utilisé à la place les estimations nationales de la prévalence. Rien ne porte à croire que les estimations de la prévalence varient considérablement dans l'ensemble du Canada, mais les études font apparaître des différences régionales dans l'utilisation des services de santé mentale $\mathrm{e}^{20}$, et ces différences influeraient sur les estimations de la prévalence des cas traités.

\section{Données administratives}

Les dossiers administratifs des utilisateurs de services de santé mentale de la zone de Calgary sont conservés dans un dépôt de données central. Tous ces utilisateurs ont été vus par un professionnel de la santé mentale (psychiatre, infirmier psychiatrique, psychologue ou travailleur social) autorisé à procéder à une évaluation diagnostique en Alberta. Pour chaque utilisateur de service, les données comprennent un identificateur unique (ULI), la source de l'aiguillage, les dates d'admission et de congé, la durée de l'hospitalisation, l'inscription à des programmes, l'âge, le sexe, le code postal, les diagnostics liés à la santé mentale reposant sur la nosologie du DSM-IV-TR et le devenir du patient au moment du congé. Les dossiers sont extraits, avec un taux de $95 \%$, des systèmes d'information sur la santé mentale utilisés pour fournir des services aux enfants, aux adultes et adolescents, aux personnes âgées et aux Autochtones, puis sont reliés dans la base de données centrale. Les $5 \%$ d'utilisateurs restants ont eu recours à des services ne permettant pas de récolter des données complètes auprès du client (p. ex. : dans certains services de crise ou d'approche, les clients ne sont pas officiellement inscrits et l'ULI n'est pas obtenu). D'après les codes postaux, la majorité des utilisateurs de services de santé mentale vivent dans la zone de Calgary.
Nous avons défini les cas de trouble bipolaire à partir de l'ensemble de données administratives en nous fondant sur les critères suivants : 1) le patient était officiellement inscrit dans un service de santé mentale de la zone de Calgary, ce service pouvant être un service en hospitalisation, un hôpital de jour, un service d'urgence psychiatrique, un service de consultation externe ou un programme d'approche dans la collectivité et 2) le diagnostic principal noté pour l'inscription du patient était un trouble bipolaire de type I ou de type II, ce diagnostic principal représentant la principale raison de l'admission du patient dans le programme en question. Cette définition de cas excluait les patients traités par d'autres travailleurs de la santé pour des soins médicaux sans rapport avec leur trouble bipolaire (p. ex. : consultation en diététique) et les rendez-vous ponctuels avec d'autres professionnels pour des questions sociales non précises (p. ex. : logement). La plupart des patients inscrits dans des services de santé mentale présentent des diagnostics multiples. La présence d'autres codes de diagnostic dans le dossier médical ne suffisait pas à exclure les patients du moment qu'un trouble bipolaire de type I ou de type II était inscrit comme diagnostic principal. Nous craignions d'inclure des diagnostics secondaires (non principaux), car il arrive souvent qu'ils soient inscrits comme diagnostic « à exclure » lors de certaines consultations. Dans tous les services de santé mentale, le diagnostic est posé en se fondant sur une évaluation clinique complète, même si les outils d'interview et autres instruments d'évaluation varient d'un programme à l'autre.

La zone de Calgary ne possède pas d'établissement psychiatrique pour des séjours de longue durée, mais il existe des établissements de soins de longue durée pour les patients âgés. Les données venant de ces établissements ne sont pas reliées au dépôt de données central des services de santé mentale. Par conséquent, les personnes âgées souffrant de trouble bipolaire qui vivent dans des maisons de soins infirmiers ne sont pas représentées dans notre estimation de la prévalence des cas traités, sauf si elles ont eu recours à l'un des services couverts. 
TABLEAU 1

Caractéristiques des patients atteints de troubles bipolaires au Canada (2002) et dans la zone de Calgary (2002-2008)

\begin{tabular}{|c|c|c|c|c|}
\hline & \multicolumn{2}{|c|}{$\begin{array}{l}\text { Canada }^{a} \\
(2002)\end{array}$} & \multicolumn{2}{|c|}{$\begin{array}{l}\text { Zone de Calgaryb } \\
(2002-2008)\end{array}$} \\
\hline & $\begin{array}{l}\text { Bipolaire I } \\
{\text { (estimation sur } 12 \text { mois })^{\mathrm{c}}}_{(\mathrm{n}=364)}\end{array}$ & $\begin{array}{l}\text { Bipolaire I } \\
\left.\text { (estimation au cours }^{\text {de la vie) }}\right)^{\mathrm{d}} \\
(\mathbf{n}=\mathbf{8 9 0})\end{array}$ & $\begin{array}{l}\text { Bipolaire I } \\
(\mathrm{n}=3659)\end{array}$ & $\begin{array}{l}\text { Bipolaire I } \\
(n=1065)\end{array}$ \\
\hline \multicolumn{5}{|c|}{ Pourcentage moyene (IC à 95 \%) } \\
\hline Hommes & $\begin{array}{c}42,2 \% \\
(35,2-49,3)\end{array}$ & $\begin{array}{c}46,1 \% \\
(41,6-50,5)\end{array}$ & $\begin{array}{c}53,7 \% \\
(52,1-55,3)\end{array}$ & $\begin{array}{c}38,5 \% \\
(35,6-41,4)\end{array}$ \\
\hline Femmes & $\begin{array}{c}57,7 \% \\
(50,7-64,8)\end{array}$ & $\begin{array}{c}53,9 \% \\
(49,5-58,4)\end{array}$ & $\begin{array}{c}46,3 \% \\
(44,6-47,9)\end{array}$ & $\begin{array}{c}61,5 \% \\
(58,6-64,4)\end{array}$ \\
\hline Âge moyen en années & $\begin{array}{c}34,8 \\
(33,0-36,5)\end{array}$ & $\begin{array}{c}38,7 \\
(37,6-39,9)\end{array}$ & $\begin{array}{c}40,0 \\
(39,5-40,5)\end{array}$ & $\begin{array}{c}39,5 \\
(38,7-40,3)\end{array}$ \\
\hline \multicolumn{5}{|c|}{ Distribution par âge (IC à 95 \%) } \\
\hline $18-24$ ans & $\begin{array}{c}26,5 \% \\
(19,9-33,1)\end{array}$ & $\begin{array}{c}17,2 \% \\
(13,5-20,9)\end{array}$ & $\begin{array}{c}17,0 \% \\
(15,8-18,2)\end{array}$ & $\begin{array}{c}14,4 \% \\
(12,3-16,5)\end{array}$ \\
\hline $25-44$ ans & $\begin{array}{c}49,9 \% \\
(42,7-57,0)\end{array}$ & $\begin{array}{c}48,0 \% \\
(43,4-52,6)\end{array}$ & $\begin{array}{c}48,4 \% \\
(46,8-50,0)\end{array}$ & $\begin{array}{c}52,0 \% \\
(49,0-55,0)\end{array}$ \\
\hline $45-64$ ans & $\begin{array}{c}23,7 \% \\
(17,7-29,6)\end{array}$ & $\begin{array}{c}33,1 \% \\
(28,8-37,4)\end{array}$ & $\begin{array}{c}27,8 \% \\
(26,3-29,2)\end{array}$ & $\begin{array}{c}29,8 \% \\
(27,0-32,5)\end{array}$ \\
\hline 65 ans et plus & $-^{f}$ & $\begin{array}{c}1,7 \% \\
(0,8-2,6)\end{array}$ & $\begin{array}{c}6,8 \% \\
(6,0-7,6)\end{array}$ & $\begin{array}{c}3,8 \% \\
(2,7-5,0)\end{array}$ \\
\hline
\end{tabular}

Abréviations : ESCC 1.2, Enquête sur la santé dans les collectivités canadiennes : Santé mentale et bien-être de 2002; IC, intervalle de confiance; n, taille de l'échantillon.

${ }^{a}$ Calculé à partir des données de l'ESCC 1.2.

bCalculé à partir des données de 2002 à 2008 du dépôt de données administratives de la zone de Calgary.

'Un ou plusieurs épisodes au cours des 12 mois précédents.

¿Un ou plusieurs épisodes au cours de la vie.

ées chiffres étant arrondis, il se peut que la somme des pourcentages ne soit pas égale à $100 \%$.

f'échantillon est trop petit pour que les données soient mentionnées; Statistique Canada interdit de publier les données fondées sur de petits échantillons afin de préserver la confidentialité des intéressés.

TABLEAU 2

Traitement par des psychiatres de troubles bipolaires de type I et de type II dans la population ayant un problème de santé mentale, zone de Calgary, 2002-2008

\begin{tabular}{|c|c|c|c|}
\hline & $\begin{array}{l}\text { Nombre d'adultes atteints d'un } \\
\text { trouble bipolaire } \\
\text { (n) }\end{array}$ & $\begin{array}{l}\text { Pourcentage de patients ayant } \\
\text { un problème de santé mentale atteints } \\
\text { d'un trouble bipolaire } \\
\text { (IC à } 95 \% \text { ) }\end{array}$ & $\begin{array}{l}\text { Prévalence des cas traités de trouble } \\
\text { bipolaire dans la zone de Calgaryb } \\
\text { en \% } \\
\text { (IC à } 95 \%)\end{array}$ \\
\hline Bipolaire I & 3659 & $\begin{array}{c}5,81 \\
(5,63-5,99)\end{array}$ & $\begin{array}{c}0,41 \% \\
(0,40-0,42)\end{array}$ \\
\hline Bipolaire II & 1065 & $\begin{array}{c}1,70 \\
(1,59-1,79)\end{array}$ & $\begin{array}{c}0,12 \% \\
(0,11-0,13)\end{array}$ \\
\hline
\end{tabular}

Abréviations : IC, intervalle de confiance; $n$, taille de l'échantillon.

aLe dénominateur est 63 016, c.-à-d. le nombre d'adultes à qui l'on a diagnostiqué un trouble mental, 2002-2008.

'Le dénominateur est 894 905, c.-à-d. la population approximative de la région sanitaire de Calgary âgée de 18 ans et plus au point médian entre 2002 et 2008.

Nous avons obtenu des estimations agrégées de la prévalence des cas traités de trouble bipolaire de l'Information and Evaluation Unit de la zone de Calgary. Ces analyses ont été effectuées " à l'interne » dans le cadre du fonctionnement de ces unités et à ce titre ne nécessitaient pas d'autorisation éthique. Les résultats tirés de la base de données administratives sont exprimés sous forme de moyenne avec des IC à $95 \%$, et ils ne sont pas pondérés, car il ne s'agit pas d'échantillons. Toutes les données que nous présentons concernent des personnes âgées de 18 ans et plus.

\section{Résultats}

Les données démographiques de base des populations étudiées sont présentées dans le tableau 1. Selon l'ESCC 1.2, 364 répondants obtiennent un résultat positif dans les algorithmes de trouble bipolaire de 
TABLEAU 3

Traitement du trouble bipolaire de type Ia par des psychiatres d'après l'ESCC 1.2, 2002, Canada

\begin{tabular}{|c|c|c|}
\hline & \multicolumn{2}{|c|}{ Estimations de la prévalence } \\
\hline & 12 mois & Au cours de la vie \\
\hline \multicolumn{3}{|l|}{ Nombre de répondants dans l'enquête, $\mathrm{n}$ : } \\
\hline Qui répondaient aux critères de trouble bipolaire de type I & $357^{c}$ & $880^{c}$ \\
\hline Qui étaient traités pour un trouble bipolaire de type I & 171 & 430 \\
\hline Pourcentage des répondants à l'ESCC 1.2 suivant un traitement psychiatrique & $\begin{array}{c}0,44 \%^{\mathrm{d}} \\
(0,36-0,52)\end{array}$ & $\begin{array}{c}1,17 \%^{\mathrm{d}} \\
(1,02-1,33)\end{array}$ \\
\hline
\end{tabular}

Abréviations : ESCC 1.2, Enquête sur la santé dans les collectivités canadiennes : Santé mentale et bien-être de 2002; IC, intervalle de confiance; n, taille de l'échantillon.

aLe trouble bipolaire de type II n'était pas inclus dans I'ESCC 1.2.

'Les nombres sont inférieurs au nombre total de répondants de l'ESCC 1.2 (36 984) à cause de données manquantes.

cLes nombres sont inférieurs à ceux qui apparaissent au tableau 1 à cause de données manquantes.

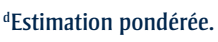

type I sur 12 mois, et 890 au cours de leur vie. La plus forte proportion de femmes tient au pourcentage plus élevé de répondantes dans l'ESCC 1.2. Cette enquête conclut que la prévalence du trouble bipolaire de type I est égale chez les hommes et les femmes ${ }^{21}$. Les données de la zone de Calgary sont très similaires à celles de l'ESCC 1.2 pour ce qui est de l'évaluation selon les critères au cours de la vie. Dans le cas de la zone de Calgary, cependant, la prévalence des cas traités est fonction du sexe. En effet, beaucoup plus d'hommes que de femmes étaient traités pour un trouble bipolaire de type I, tandis que l'inverse était vrai pour le trouble bipolaire de type II, près des deux tiers des patients traités étant des femmes. Cet écart fait apparaître une différence entre les troubles sur le plan de la recherche d'aide, selon le sexe.

La stratification par groupe d'âge (tableau 1) montre que les quatre populations étudiées étaient similaires pour ce qui est de la distribution par âge. La seule exception manifeste est celle de la population sensiblement plus jeune dont les résultats sont positifs pour les épisodes de trouble bipolaire de type I dans les 12 derniers mois dans l'ESCC 1.2 en comparaison avec les trois autres groupes.

Pour évaluer la prévalence des cas traités de troubles bipolaires dans la zone de Calgary, nous avons utilisé les données administratives, ce qui nous a donné 0,41 \% pour le trouble de type I et $0,12 \%$ pour celui de type II (tableau 2).

Les données de l'ESCC 1.2 nous ont permis d'évaluer la proportion de Canadiens souffrant de troubles bipolaires de type I qui se font soigner. Le trouble bipolaire de type II n'était pas visé par l'enquête. Nous avons fait des estimations des épisodes au cours des 12 derniers mois et au cours de la vie, et nous nous attendions à ce qu'elles encadrent les estimations faites à partir des données administratives portant sur sept ans. Elles ont été de $0,44 \%$ pour les épisodes au cours des 12 derniers mois et de $1,17 \%$ pour ceux au cours de la vie (tableau 3 ).

\section{Analyse}

Nous sommes les premiers, à notre connaissance, à étudier la concordance entre les taux de traitement autodéclarés et les dossiers administratifs comme tels pour un trouble de santé mentale particulier.

Un élément clé de cette étude est l'utilisation du dépôt de données plutôt que des données de facturation des médecins. Nos résultats montrent que l'estimation fondée sur l'enquête en population de la proportion de personnes souffrant d'un trouble bipolaire qui déclarent elles mêmes être traitées par un psychiatre est proche de l'estimation de la prévalence des cas traités découlant des dossiers administratifs des utilisateurs des services de santé mentale. La concordance de ces estimations est une constatation importante, qui a des conséquences pour les études de prévalence futures. En effet, il pourrait se révéler économique d'utiliser les données administratives et ce serait un moyen accessible d'évaluer avec précision la prévalence d'un trouble dans l'ensemble de la population.

Comme nous n'avons pas pu tenir compte des patients traités par un MG et non par un psychiatre, il faudrait savoir quelle proportion de patients de la zone de Calgary est soignée uniquement par un MG. En utilisant les données de l'ESCC 1.2 sur les répondants dont il est déterminé qu'ils souffrent d'un trouble bipolaire de type I, nous avons estimé que la prévalence de ce trouble au cours de la vie chez les répondants âgés de 18 ans et plus s'établit à 2,39\% (IC à $95 \%: 2,19 \%$ à 2,60\%) ) $^{\ddagger}$ et que la proportion de personnes traitées par un MG seulement est de 0,46\% (IC à $95 \%$ : 0,35 \% à $0,57 \%)$. En réalité, une plus forte proportion de répondants $(1,17 \%$; tableau 3$)$ reçoit des soins psychiatriques et, par conséquent, la proportion de patients recevant ce type de soins serait de $72 \%$ : [1,17/ $(1,17+0,46)] \times 100$. Il semble donc que

${ }^{\ddagger}$ Ce qui est légèrement différent de la prévalence de 2,2 \% mentionnée par Shaffer et coll. ${ }^{21}$, parce que leur résultat portait sur les répondants âgés de 15 ans et plus. 
le dépôt de données contienne les dossiers de la majorité (environ $70 \%$ ) des patients recevant des soins médicaux pour un trouble bipolaire de type I dans la région sanitaire de Calgary.

Quelle proportion de patients souffrant d'un trouble bipolaire n'est soignée ni par un MG ni par un psychiatre? Nous estimons, d'après l'ESCC 1.2, que 0,73\% (IC à $95 \%: 0,62 \%$ à $0,84 \%$ ) des répondants souffrant d'un trouble bipolaire de type I ne reçoivent pas de soins médicaux. Les personnes atteintes d'une forme légère de trouble bipolaire n'ont sans doute pas besoin de traitement. Cependant, certaines présentent des troubles importants d'un point de vue clinique, et gagneraient probablement à être traitées, mais ne cherchent pas à se faire soigner de peur d'être stigmatisées ou parce que l'accès à des soins spécialisés est limité. Ces situations sont évidemment lourdes de conséquences, mais il est probable que l'existence de diverses sources d'information aidera à établir une distinction entre ces possibilités. Les données des enquêtes permettent d'évaluer la proportion d'une population souffrant d'un trouble diagnosticable, tandis que la prévalence des cas traités se limite à la proportion de la population recevant de fait un traitement. Ces résultats montrent que les données administratives offrent sans doute une perspective fort utile de la prévalence des cas traités de trouble bipolaire.

L'une des limitations des enquêtes sanitaires est qu'elles reposent sur l'autodéclaration. Les données administratives, elles, fournissent une évaluation objective du traitement reçu. Dans le cas des troubles mentaux relativement peu fréquents dans la population, elles peuvent fournir nettement plus de cas pour les analyses que les échantillons d'enquête ${ }^{3}$. Ce constat est évident dans notre étude : l'échantillon de cas de trouble bipolaire de type I obtenu à partir des sources de données administratives était nettement supérieur à celui de l'enquête nationale sur la santé mentale (tableau 1).

Il arrive que les chercheurs mettent en doute la qualité des données administratives, en particulier en ce qui concerne le codage des diagnostics ${ }^{22,23}$. Des études locales de seconde saisie des données relativement aux rencontres avec les patients hospitalisés ${ }^{24,25}$ laissent supposer que les méthodes de codage de la zone de Calgary sont fiables. Bien que les taux de sensibilité varient considérablement d'un trouble médical à l'autre, les taux de spécificité à Calgary sont de $99 \%$ ou mieux pour tous les troubles examinés (c.-à-d. dans presque tous les cas, le diagnostic principal inscrit au dossier pour la rencontre avec le patient hospitalisé a été vérifié par un expert médical indépendant). Nous reconnaissons qu'il y a peu de recherches sur la validité des diagnostics de santé mentale dans les données administratives.

Il est à noter que nous avons peut-être surestimé la prévalence réelle des cas traités, car il arrive que certaines personnes contactent un médecin, mais ne reçoivent pas de traitement. C'est pourquoi le terme " prévalence des contacts » serait sans doute préférable lorsque l’on évalue la prévalence d'une maladie à partir de sources de données administratives ${ }^{26}$.

\section{Limites}

Une des limites de notre étude tient à ce que nous n'avons pas pu évaluer la proportion de patients bipolaires traités par les psychiatres du secteur privé (environ $30 \%$ ) qui ne sont pas rattachés aux services psychiatriques de la zone de Calgary. Cela signifie probablement que la prévalence réelle des troubles bipolaires traités par des psychiatres dans la zone de Calgary (tableau 2) est encore plus proche des estimations nationales réalisées à partir des enquêtes (tableau 3).

De plus, l'ESCC 1.2 ne comprend pas les peuples autochtones et les personnes vivant en établissement. Comme il est impossible d'enlever ces deux catégories du dépôt de données, la comparaison entre les données administratives et les données de l'ESCC 1.2 est limitée.

L'ESCC 1.2 est également limitée par le fait que les critères retenus pour le trouble bipolaire de type I ne sont pas tout à fait conformes aux critères du DSM-IV. Selon ces derniers, des symptômes maniaques doivent être présents pendant au moins une semaine ou toute autre durée si une hospitalisation est nécessaire ${ }^{15}$. Quant aux critères de l'ESCC 1.2, les symptômes maniaques doivent être présents pendant quatre jours, ce qui réduit la spécificité par rapport à celle que l'on peut obtenir en appliquant strictement les critères du DSM-IV. Cette considération explique sans doute en partie l'estimation plus élevée du traitement du trouble bipolaire de type I à partir des données de l'ESCC 1.2 par rapport à celle réalisée à partir de nos données administratives locales.

En résumé, nous avons constaté un degré de concordance important entre les estimations des troubles bipolaires de type I traités dans les données administratives locales et les données de l'enquête nationale. Cette observation renforce l'idée que les dépôts de données administratives peuvent être utiles dans la surveillance des troubles mentaux chroniques.

\section{Remerciements}

Ce projet a été financé par une subvention du Hotchkiss Brain Institute de la faculté de médecine de l'Université de Calgary. Nous remercions M. Jim Si (Population Surveillance Group, Calgary Zone) pour son estimation de la population de la zone de Calgary en 2005. Les données de l'ESCC 1.2 ont été recueillies par Statistique Canada. Cependant, les analyses et interprétations présentées ici sont celles des auteurs et non celles de Statistique Canada.

\section{Références}

1. Enquête sur la santé dans les collectivités canadiennes : santé mentale et bien-être 2002 [Internet]. Ottawa (Ont.) : Santé Canada; 2003 [Consultation le 6 avril 2010] Consultable en ligne à la page : http://www .statcan.gc.ca/pub/82-617-x/index-fra.htm

2. Frohlich KL, Dunn JR, McLaren L, Shiell A, Potvin L, Hawe P, et coll. Understanding place and health: a heuristic for using administrative data. Health Place. 2007;13:299-309.

3. Mortensen PB. The untapped potential of case registers and record-linkage studies in psychiatric epidemiology. Epidemiologic Rev. 1995;17:205-9.

4. Comité consultatif sur la santé de la population et la sécurité de la santé, Groupe de travail sur les systèmes de surveillance des facteurs de risque reliés aux maladies chroniques. 
Accroître la capacité de surveillance des facteurs de risque et des déterminants reliés aux maladies chroniques, Ottawa (Ont.) : Agence de santé publique du Canada; 2005. N $N^{o}$ de catalogue HP5-11/2005.

5. Greenberg GA, Rosenheck RA. Does system reform reduce geographic variation in mental health system performance. Psychiatric Q. 2005;76:231-42.

6. Speer DC, Newman FL. Mental health services outcome evaluation. Clin Psychol Sci Pr. 1996;3:105-29.

7. Addington D, McKenzie E, Addington J, Patten S, Smith H, Adair C. Performance measures for early psychosis treatment service. Psychiatric Serv. 2005;56:1570-82.

8. Karlin BE, Norris MP. Public mental health care utilization by older adults. Adm Policy Ment Health. 2006;33:730-6.

9. Andrews G, Issakidis C, Sanderson K, Corry J, Lapsley H. Utilising survey data to inform public policy: comparison of the cost-effectiveness of treatment of ten mental disorders. Br J Psychiatry. 2004;184:526-33.

10. Slomp M, Bland R, Patterson S, Whittaker L. Three-year physician treated prevalence rate of mental disorders in Alberta. Can J Psychiatry. 2009;54:199-203.

11. Andrews G. It would be cost-effective to treat more people with mental disorders. Aust N Z J Psychiatry. 2006;40:613-5.

12. Paradis M, Woogh C, Marcotte D, Chaput Y. Is psychiatric emergency service (PES) use increasing over time? Int $\mathrm{J}$ Ment Health Syst. 2009;3:3.

13. Woogh CM. An experience in psychiatric record linkage. Can J Psychiatry. 1988;33:134-9.

14. Oswald P, Souery D, Kasper, Lecrubier Y, Montgomery S, Wyckaert S, et coll. Current issues in bipolar disorder: a critical review. Eur Neuropsychopharmacol. 2007;17:687-95.

15. American Psychiatric Association. Manuel diagnostique et statistique des troubles mentaux, quatrième éd., texte révisé. Paris : Masson; 2003.
16. Zimmerman M, Ruggero CJ, Chelminski I, Young D. Is bipolar disorder overdiagnosed? J Clin Psychiatry. 2008;69:935-40.

17. Patten SB, Paris J. The bipolar spectrum-a bridge too far? Can J Psychiatry. 2008;53:762-8.

18. Gravel R, Beland Y. The Canadian Community Health Survey: mental health and well-being. Can J Psychiatry. 2005;10:573-9.

19. Kessler RC, Ustun TB. The World Mental Health (WMH) Survey Initiative version of the World Health Organization (WHO) Composite International Diagnostic Interview (CIDI). Int $\mathrm{J}$ Methods Psychiatr Res. 2004;13:93-121.

20. Diaz-Granados N, Georgiades K, Boyle MH. Regional and individual influences on use of mental health services in Canada. Can J Psychiatry. 2010;55:9-20.

21. Schaffer A, Cairney J, Cheung A, Veldhuizen S, Levitt A. Community survey of bipolar disorder in Canada: lifetime prevalence and illness characteristics. Can J Psychiatry. 2006;51:9-16.

22. Roos LL, Soodeen R, Gupta S, Jebamani L. Canadian administrative data: evaluating the quality. Winnipeg (MB): University of Manitoba; 2002.

23. Roos LL, Gupta S, Soodeen R, Jebamani L. Data quality in an information-rich environment: Canada as an example. Can J Ageing. 2005;24, Suppl 1:153-70.

24. Quan H, Parsons GA, Ghali WA. Validity of procedure codes in International Classification of Diseases, 9th revision, clinical modification of administrative data. Med Care. 2004;42:801-9.

25. Quan H, Parsons GA, Ghali WA. Assessing accuracy of diagnosis-type indicators for flagging complications in administrative data. J Clin Epidemiol. 2004;57:366-74.

26. Goldner EM, Jones W, Waraich P. Using administrative data to analyze the prevalence and distribution of schizophrenic disorders. Psychiatr Serv. 2003;54:1017-21. 